\title{
Endophthalmitis caused by Acinetobacter baumanni: a case series
}

\begin{abstract}
Purpose To profile the etiology, clinical outcomes and drug sensitivity patterns in endophthalmitis caused by Acinetobacter baumanni.

Methods Retrospective analysis of all the cases of Acinetobacter baumanni endophthalmitis presenting to tertiary referral care ophthalmic hospital in Eastern India from January 2009 to December 2011 were done. Results A total of four cases were included in the study. Out of the four cases one was post traumatic and the rest were post cataract surgery. All the cases underwent vitreoretinal surgical intervention followed by intravitreal antibiotics. A. Baumanni was isolated from vitreous in all the cases. Among all the drugs tested bacteria were found sensitive to ciprofloxacin $(100 \%)$ whereas all tested resistant to ceftazidime. Out of the four cases one had to be eviscerated, another developed retinal detachment post vitrectomy, one was phthisical at final followup, and only one patient achieved a vision of 20/200 with clear media and attached retina at final visit. Conclusion A. Baumanni is a very rare cause of endophthalmitis with poor visual and anatomical outcomes. Ciprofloxacin should be considered as first the line intravitreal antibiotic.
\end{abstract}

R Roy', P Panigrahi', J Malathi², SS Pal', K Nandi', A Patil ${ }^{1}$, E Nigam ${ }^{1}$ and $V$ Arora' with only a single report available in literature. ${ }^{1}$ Purpose of this study is to report the clinical profile and drug sensitivity patterns of A. baumanni endophthalmitis.

Case reports

Case 1

A 60-year-old lady presented with pain, redness, and loss of vision in her right eye 2 days after phacoemulsification. Visual acuity was light perception (PL). Examination revealed corneal abscess with exudates in anterior chamber. Ultrasound revealed dense vitreous echoes with attached retina. She underwent vitrectomy with intraocular lens (IOL) removal and intravitreal antibiotics (vancomycin: $1 \mathrm{mg} / 0.1 \mathrm{ml}$, ceftazidime: $2.25 \mathrm{mg} / 0.1 \mathrm{ml}$, and dexamethasone: $0.4 \mathrm{mg}$ / $0.1 \mathrm{ml}$ ). In spite of surgery, her condition worsened and she developed panophthalmitis. Finally she underwent evisceration.

Case 2

A 48-year-old male patient presented with pain, redness, and loss of vision in his left eye 3 days after cataract surgery. At presentation, vision was PL. Examination revealed superior scleral wound dehiscence with iris prolapse. Cornea was oedematous with hypopyon and membrane over the IOL. Ultrasound revealed attached retina with dense vitreous echoes. He underwent wound resuturing, excision of the prolapsed iris, removal of IOL, vitrectomy, and intravitreal antibiotics. He developed retinal detachment at the 7th post-operative day. $\mathrm{He}$ was advised resurgery, which he declined.

Case 3

A 74-year-old lady presented with pain and redness in her right eye 15 days after cataract

\footnotetext{
Eye (2013) 27, 450-452; doi:10.1038/eye.2012.277; published online 11 January 2013

Keywords: retina; endophthalmitis; Acinetobacter

Introduction

Endophthalmitis is a visually devastating condition having poor clinical outcome in majority of the cases. Amongst other factors, the nature of the infective strain has got a profound bearing on the final result. Acinetobacter baumanni is a rare cause of endophthalmitis

\author{
Aditya Birla Sankara
Nethralaya, Kolkata,
}
Received: 1 August 2012 Accepted in revised form
Fax: +913344013199.
E-mail: rayrupak@
gmail.com
19 November 2012
Microbiology, L\&T
Microbiology Research
Centre, Sankara Neth
Chennai, India
Correspondence:
R Roy, Vitreo Retina
Services, Aditya Birla
Sankara Nethralaya,
E.M.Bypass, Kolkata
700099, West Benga
Microbiology, L\&T
Microbiology Research
Centre, Sankara Neth
Chennai, India
Correspondence:
R Roy, Vitreo Retina
Services, Aditya Birla
Sankara Nethralaya, 1
E.M.Bypass, Kolkata
700099, West Bengal
Microbiology, L\&T
Microbiology Research
Centre, Sankara Neth
Chennai, India
Correspondence:
R Roy, Vitreo Retina
Services, Aditya Birla
Sankara Nethralaya, 1
E.M.Bypass, Kolkata
700099, West Bengal, India.


Table 1 Salient clinical details

\begin{tabular}{lcccccc}
\hline Case no & Age/gender & Aetiology & Presentation & BCVAI & Intervention & BCVAF \\
\hline 1 & $60 / \mathrm{F}$ & Post cat sx & Fulminant & $\mathrm{PL}$ & $\mathrm{V}+$ intravit Ab & No PL \\
2 & $48 / \mathrm{M}$ & Post cat sx & Fulminant & $\mathrm{PL}$ & $\mathrm{V}+$ intravit Ab & PL \\
3 & $74 / \mathrm{F}$ & Post cat sx & Acute & $\mathrm{PL}$ & $\mathrm{V}+$ intravit Ab & $20 / 200$ \\
4 & $7 / \mathrm{F}$ & Post traumatic & Acute & PL & L $+\mathrm{V}+$ intravit Ab & No PL \\
\hline
\end{tabular}

Abbreviations: Ab, antibiotics; Acute, $>4$ days and $<6$ weeks; BCVAF, final best corrected visual acuity; BCVAI, best corrected visual acuity at presentation; Cat Sx, cataract surgery; F, Female; Fulminant, <4 days, L= lensectomy; M, male; PL, Perception of light; V, vitrectomy.

Table 2 Microbiological details and drug sensitivity patterns

\begin{tabular}{lccccccccc}
\hline Case no & Aspirate & Gm stain & PCR & Culture & Cefta & Ami & Cipro & Genta & Cepho \\
\hline 1 & $\mathrm{~V}$ & $\mathrm{~N}$ & Eubac & A. baumanni & $\mathrm{R}$ & $\mathrm{S}$ & $\mathrm{S}$ & $\mathrm{S}$ & $\mathrm{S}$ \\
2 & $\mathrm{~V}$ & $\mathrm{~N}$ & Eubac & A. baumanni & $\mathrm{R}$ & $\mathrm{S}$ & $\mathrm{S}$ & $\mathrm{S}$ & $\mathrm{R}$ \\
3 & $\mathrm{~V}$ & $\mathrm{~N}$ & Eubac & A. baumanni & $\mathrm{R}$ & $\mathrm{R}$ & $\mathrm{S}$ & $\mathrm{R}$ & $\mathrm{S}$ \\
4 & $\mathrm{~V}$ & Gm negative bacilli & Eubac & A. baumanni & $\mathrm{R}$ & $\mathrm{S}$ & $\mathrm{S}$ & $\mathrm{S}$ & $\mathrm{R}$ \\
\hline
\end{tabular}

Abbreviations: Ami, amikacin; Cefta, ceftazidime; Cepho, cephotaxime; Cipro, ciprofloxacin; Eubac, eubacterial genome positive; Genta, gentamycin; Gm, gram; N, no result; PCR, polymerase chain reaction; R, resistant; S, sensitive; V, vitreous.

surgery. Visual acuity was PL. Cornea was oedematous with hypopyon and exudative membrane over IOL. Ultrasound showed dense vitreous echoes with attached retina. She underwent vitrectomy with IOL removal and intravitreal antibiotics. Intraoperatively, a subretinal abscess was noted at the macular area. In the postoperative period, the patient was on alternate day regimen of intravitreal antibiotics. At final followup, disc was pale with a scar at the macular area. Visual acuity improved to $20 / 200$.

\section{Case 4}

A 7-year-old girl presented 19 days after penetrating trauma with needle to her right eye. Examination revealed a sutured corneal tear with exudates in the pupillary area. Ultrasound revealed dense vitreous echoes with attached retina. She underwent lensectomy, vitrectomy, and intravitreal antibiotics. Intraoperatively, retina was found to be ischaemic with a pale disc. At final followup, the eye was phthisical.

Salient details of the cases are summarised in Table 1.

\section{Discussion}

Acinetobacter are nonmotile, oxidase negative gram negative bacilli classified under family Moraxcellaceae. ${ }^{2}$ A baumanni is an important member of this group, which causes a variety of human clinical infections involving skin, soft tissue, and bone. ${ }^{3}$ Recently, it has emerged as an important cause of community-acquired infections. It has gained notoriety for its ability to upregulate and acquire resistance to antibiotics. ${ }^{2}$ Endophthalmitis caused by A baumanni is rare. English language literature review reveals only a single report of two cases of $A$. baumanni endophthalmitis. To the best of our knowledge, this is the second report on A. baumanni endophthalmitis. In the present series, the organism A. baumanni was isolated from all cases in more than one media. The bacteria were identified to species level by application of standard biochemical reactions. Antibiotic-disc diffusion test was carried out according to CSLI guidelines. Chen et al ${ }^{1}$ has reported two cases of $A$. baumanni endophthalmitis. Out of those two cases, one was endogenous and the other was post keratoplasty. Though A. baumanni is an important cause of nosocomial infections, none of our cases were endogenous. All our cases had an early presentation, indicating the virulence of the organism. In spite of aggressive therapy, all but one had very poor outcome. Similarly, Chen et al ${ }^{1}$ reports visual acuity outcomes of 20/60 and no LP in their two cases. Antimicrobial resistance of Acinetobacter is of serious concern. Among all the drugs tested, bacteria were found sensitive to ciprofloxacin $(100 \%)$, whereas all tested resistant to ceftazidime (Table 2). Gopal et al has also reported high level of sensitivity to ciprofloxacin exhibited by Acinetobacter species. Chen et al ${ }^{1}$ has reported sensitivity to imipenem of their isolates, however, due to logistical reasons, we were unable to test sensitivity to imipenem. In non-responding cases and where resistance to ceftazidime is seen, ciprofloxacin can be used as an intravitreal antibiotic. To conclude, $A$. baumanni is a rare cause of endophthalmitis with mostly poor visual outcome. 
What was known before

- Acinetobacter baumanni is a rare cause of endophthalmitis.

What this study adds

- Adds to the body of litearture of endophthalmitis caused by this organism. Provides drug sensitivity results that can be used for treatment.

\section{Conflict of interest}

The authors declare no conflict of interest.

\section{Acknowledgements}

This research received no specific grant from any funding agency in the public, commercial or not-for-profit sectors.

\section{References}

1 C Kuan-Jen, H Chiun-Ho, S Ming-Hui, L Chi-Chun, S Chi-Chin, H Ching-Hsi. Endophthalmitis caused by Acinetobacter baumannii: report of two cases. J Clin Microbiol 2008; 46(3): 1148-1150.

2 Peleg Anton Y, S Harald, LP David. Acinetobacter baumannii: emergence of a successful pathogen. Clin Microbiol Rev 2008; 21(3): 538-582.

3 Hsueh PR, Teng LJ, Chen CY, Chen WH, Yu CJ, Ho SW et al. Pandrug-resistant Acinetobacter baumannii causing nosocomial infections in a university hospital. Taiwan Emerg Infect Dis 2002; 8: 827-832.

4 Gopal L, Ramaswamy AA, Madhavan HN, Battu RR, Sharma T, Shanmugam MP et al. Endophthalmitis caused by Acinetobacter calcoaceticus: a profile. Indian J Ophthalmol 2003; 51: 335-340. 